\title{
The effect of tranexamic acid on the risk of death and hysterectomy in women with post-partum haemorrhage: statistical analysis plan for the WOMAN trial
}

Haleema Shakur ${ }^{1 * \dagger}$, lan Roberts ${ }^{1 \dagger}$, Philip Edwards ${ }^{1+}$, Diana Elbourne ${ }^{1}$, Zarko Alfirevic ${ }^{2}$ and Carine Ronsmans ${ }^{3}$

\begin{abstract}
Background: Severe haemorrhage is a leading cause of maternal death worldwide. Most haemorrhage deaths occur soon after childbirth. Severe post-partum bleeding is sometimes managed by the surgical removal of the uterus (hysterectomy). Death and hysterectomy are important health consequences of post-partum haemorrhage, and clinical trials of interventions aimed at preventing these outcomes are needed.

Methods: The World Maternal Antifibrinolytic trial aims to determine the effect of tranexamic acid on death, hysterectomy and other health outcomes in women with post-partum haemorrhage. It is an international, multicentre, randomised trial. Approximately 20,000 women with post-partum haemorrhage will be randomly allocated to receive an intravenous injection of either tranexamic acid or matching placebo in addition to usual care. The primary outcome measure is a composite of death in hospital or hysterectomy within 42 days of delivery. The cause of death will be described. Secondary outcomes include death, death due to bleeding, hysterectomy, thromboembolic events, blood transfusion, surgical and radiological interventions, complications, adverse events and quality of life. The health status and occurrence of thromboembolic events in breastfed babies will also be reported. We will conduct subgroup analyses for the primary outcome by time to treatment, type of delivery and cause of haemorrhage. We will conduct an analysis of treatment effect adjusted for baseline risk.
\end{abstract}

Discussion: The World Maternal Antifibrinolytic trial should provide reliable evidence for the efficacy of tranexamic acid in the prevention of death, hysterectomy and other outcomes that are important to patients. We present a protocol update and the statistical analysis plan for the trial.

Trial registration: Current Controlled Trials ISRCTN76912190 (Registration date 08 December 2008), Clinicaltrials.gov NCT00872469 (Registration date 30 March 2009) and Pan African Clinical Trials Registry: PACTR201007000192283 (Registration date 02 September 2010).

Keywords: Post-partum haemorrhage, Tranexamic acid, Clinical trial, Statistical analysis plan

\footnotetext{
* Correspondence: Haleema.Shakur@Lshtm.ac.uk

This reply refers to the comment available at: http://

trialsjournal.biomedcentral.com/articles/10.1186/1745-6215-11-40.

${ }^{\dagger}$ Equal contributors

${ }^{1}$ Clinical Trials Unit, London School of Hygiene \& Tropical Medicine, Keppel

Street, London WC1E 7HT, UK

Full list of author information is available at the end of the article
} 


\section{Introduction}

Severe haemorrhage is associated with approximately onequarter of maternal deaths worldwide [1]. Most of these deaths occur soon after childbirth. Other leading causes of maternal death are pre-existing medical conditions, high blood pressure and sepsis [2]. Severe post-partum bleeding is sometimes managed by the surgical removal of the uterus (hysterectomy). As well as the morbidity from the surgery, hysterectomy can cause psychological distress, particularly in women who want to have more children [3]. Death and hysterectomy are important health consequences of postpartum haemorrhage (PPH), and clinical trials of interventions aimed at preventing these outcomes are needed.

Tranexamic acid (TXA) reduces bleeding by inhibiting the enzymatic breakdown of the blood-clotting proteins fibrinogen and fibrin. A systematic review of clinical trials of TXA in surgery showed that TXA reduces blood loss by approximately one-third [4]. TXA also reduces mortality in bleeding trauma patients. When given soon after injury, TXA reduces the risk of death due to bleeding by approximately one-third $[5,6]$. A randomised, controlled, open-label trial of TXA in women with PPH showed that TXA reduced blood loss but recommended that a larger international study is needed to investigate whether TA can decrease the need for invasive procedures and reduce maternal morbidity in women with $\mathrm{PPH}$ [7]. Also, a systematic review of TXA for the prevention and treatment of $\mathrm{PPH}$ concluded that insufficient evidence exists to support its use [8]. Taken together, reason exists to believe that TXA might reduce deaths due to bleeding following childbirth.

The World Maternal Antifibrinolytic (WOMAN) trial aims to determine the effect of TXA on death, hysterectomy and other health outcomes in women with postpartum haemorrhage. The trial protocol was published before the start of the trial [9]. The planned sample size was increased from 15,000 to 20,000 participants in May 2014. This paper gives the reason for this increase and presents the statistical analysis plan. Recruitment of 20,000 women should be complete by the summer of 2016. The statistical analysis plan was completed before the treatment allocation was un-blinded.

\section{Methods}

\section{Study design and patients}

The WOMAN trial is an international, multicentre, randomised, placebo-controlled trial. Adult women with post-partum haemorrhage following vaginal or caesarean section delivery who have a clinical diagnosis of postpartum haemorrhage are eligible for enrolment. The clinical diagnosis of PPH may be based on blood loss of over $500 \mathrm{~mL}$ after vaginal delivery or $1,000 \mathrm{~mL}$ after caesarean section or blood loss sufficient to compromise the haemodynamic stability. The fundamental eligibility criterion is the responsible clinician's 'uncertainty' as to whether or not to use TXA in a particular woman with post-partum haemorrhage. Where TXA is indicated or was administered, or where it is contra-indicated, patients are not included in the trial. No pre-specified exclusion criteria exist. Patients receive all routine clinically indicated medical care and additionally are randomised to receive TXA or placebo. Hospitals where TXA is in routine use for PPH are not included in the trial. As the eligibility criteria automatically excluded patients who had received TXA, specific data on whether it was used before enrolment was not collected.

\section{Randomisation and masking}

Randomisation codes were generated and secured by an independent statistical consultant from Sealed Envelope Ltd (London, UK). Trial treatment packs are prepared in accordance with the randomisation list. Women eligible for inclusion are randomised to receive either active (TXA) or placebo (sodium chloride $0.9 \%$ ) intravenously. Baseline information is collected on the trial entry form, and the next lowest consecutively numbered pack is taken from a box of eight treatment packs. Once the treatment ampoule is confirmed as intact, the patient is considered to be randomised. Once a patient has been randomised, the outcome for the woman is obtained even if the trial treatment is interrupted or not given. Both participants and study staff (site investigators and trial coordinating centre staff) are masked to treatment allocation. An emergency un-blinding service is available.

\section{Trial procedures}

After eligibility is confirmed and the appropriate consent procedure followed, each patient is assigned a uniquely numbered treatment pack. In this way they are randomly allocated to receive 1 gram of TXA or placebo by intravenous injection. If bleeding continues after 30 minutes, or if bleeding stops and restarts within $24 \mathrm{~h}$, a second dose of $1 \mathrm{~g}$ of TXA or placebo may be given. No preestablished maximum time between the start of $\mathrm{PPH}$ and administration of the study treatment exists. After a patient has been randomised, the outcome in hospital is collected even if the trial treatment is interrupted or is not actually given. Outcome is collected at 6 weeks (42 days, as this usually defines the post-partum period) after randomisation, at discharge from the randomising hospital or at death (whichever occurs first). Adverse events are reported in-hospital and after discharge up to day 42 .

\section{Ethics approval}

Before the trial can start at a site, all relevant regulatory and ethics approvals are obtained. An additional file 
presents the current list of ethics approval for participating countries and sites (see Additional file 1).

\section{Consent}

The trial is being conducted in accordance with the International Conference on Harmonisation of Technical Requirements for Registration of Pharmaceuticals for Human Use-Good Clinical Practice (ICH-GCP), and participant consent is obtained in accordance with the approved protocol. The consent procedure used in the WOMAN Trial is detailed in the published protocol [9]. The informed consent procedure used at each site is approved by the relevant ethics and regulatory agencies. In summary, consent is obtained from patients if physical and mental capacity allows consent. If a patient cannot give consent, proxy consent is obtained from a relative or representative. If a proxy was unavailable, then if permitted by local regulation, consent is deferred or waived. When consent is deferred or given by a proxy, the patient is informed about the trial as soon as possible, and consent obtained for use of the data collected, if needed.

\section{Sample size}

Before the trial started, we anticipated a baseline event rate of $2.5 \%$ for death and $2.5 \%$ for hysterectomy. Assuming a control group event rate of $2.5 \%$ for death and $2.5 \%$ for hysterectomy and that $1 \%$ of women die after hysterectomy, a study with 15,000 women should have more than $90 \%$ power to detect a $25 \%$ reduction from
$4 \%$ to $3 \%$ in the primary endpoint of death or hysterectomy at the $5 \%$ significance level. Expected loss to follow-up (less than $1 \%$ ) should not impact importantly on the power (at the time of writing this Statistical Analysis Plan, we had achieved $99 \%$ follow-up at 42 days after randomisation, at discharge from hospital or death).

However, because the case fatality observed in the trial (by December 2013) was higher (3\%) than expected, a modest increase in the sample size from 15,000 to 20,000 women would mean that the trial may have sufficient (90 \%) power to detect a $25 \%$ reduction in maternal mortality alone at the $5 \%$ significance level. This increase also provided additional power to detect an effect on the composite endpoint of death or hysterectomy, whilst providing protection against the possibility that the effect of TXA on death and hysterectomy is different. Increasing the sample size to 20,000 women involved an extra 15 months of recruitment.

The primary outcome remains the same (i.e. a composite of death and hysterectomy) with death alone as a secondary outcome. The independent Data Monitoring Committee was aware of the higher case fatality, as these data were presented as part of their routine review. The independent Trial Steering Committee also reviewed and approved the sample size increase.

\section{Statistical analysis}

\section{Trial profile}

The flow of study participants will be displayed in a CONSORT diagram [10], as shown in Fig. 1. Because

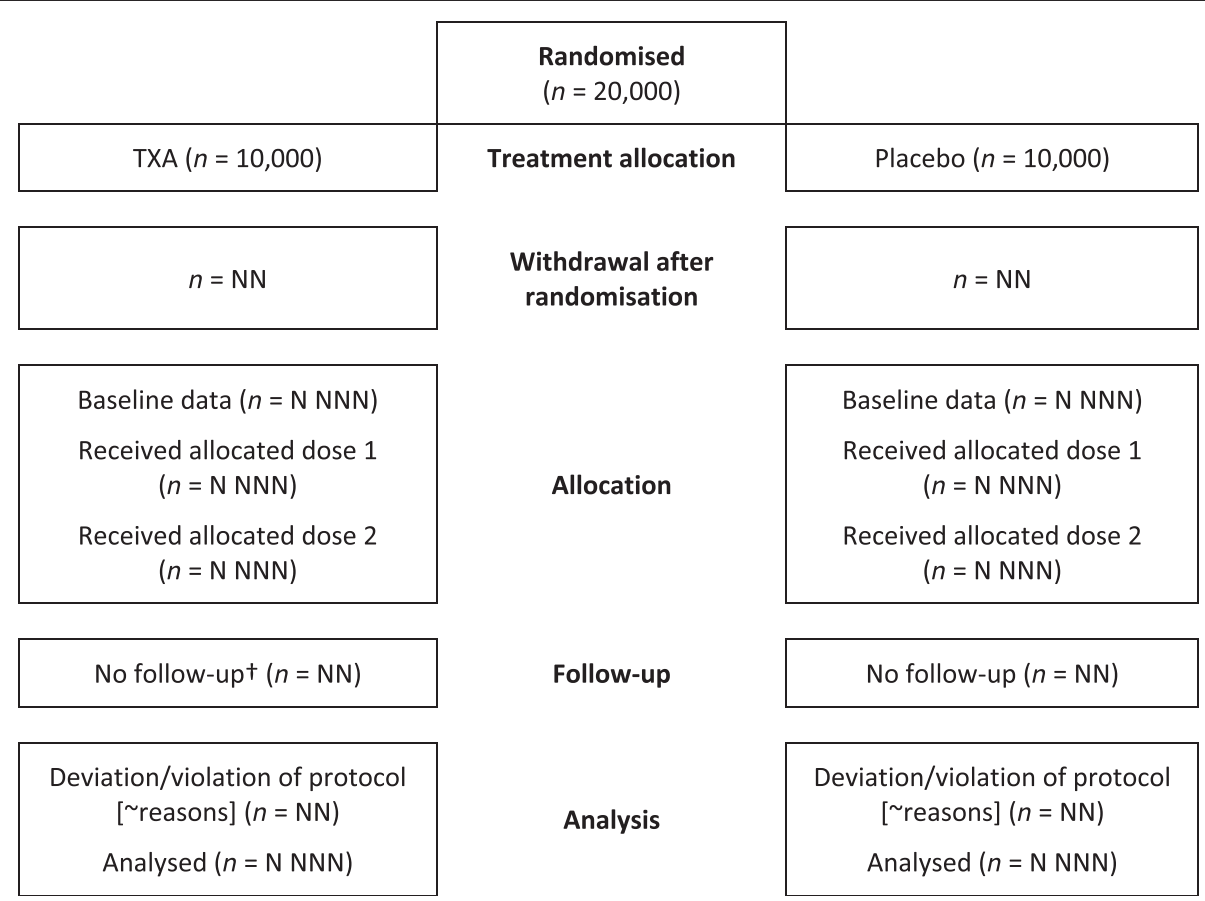

Fig. 1 Trial profile. ${ }^{\dagger}$ No follow-up relates to those patients where there is no information on the primary endpoint 
the trial involves a life-threatening emergency situation, we did not require clinicians to complete a screening $\log$. The number of patients included in the primary and secondary analyses, the reasons for exclusions and the number of patients lost to follow-up will be reported.

Patients randomised who did not fulfil the eligibility criteria and those who did not receive their allocated treatment will be considered as having deviated from the protocol. Their data will be included in the intention-totreat (ITT) analysis. If a patient or their representative withdraws consent for data collection, only data up to the point of withdrawal will be used in the analysis. Additionally, we will summarise the type of consent process used (number and percentage): patient, personal representative, professional representative and waiver.

\section{Planned analysis}

\section{Primary analysis}

The main analyses will compare all those allocated tranexamic acid versus those allocated placebo, on an 'intention-to-treat' basis, irrespective of whether they received the allocated treatment or not.

\section{Baseline characteristics}

To check that randomisation produced broadly similar groups, the baseline characteristics obtained prior to randomisation will be presented as the number and percentage in each group (Table 1).

\section{Primary outcome: death or hysterectomy}

The primary outcome is a composite of death or hysterectomy. Death and hysterectomy are important outcomes for women, are easily measured and could potentially be prevented by TXA. We use this composite endpoint to increase the number of outcome events, thereby increasing the statistical power to detect a treatment effect [11]. Although the increase in power comes at a cost of greater uncertainty in interpretation of the results, the use of a composite endpoint is justified if the components are of similar importance, occur with similar frequency and are likely to be affected to a similar degree by the treatment $[12,13]$. Whilst hysterectomy cannot be considered as important as death, the composite may be justified on the basis that hysterectomy is conducted as a last resort to prevent death from bleeding, in which case this less serious outcome may be a reasonable surrogate for maternal death.

We anticipated that death and hysterectomy would occur with similar frequency. Whether death and hysterectomy will be affected to a similar degree by TXA is unknown, but to the extent that death and hysterectomy are direct consequences of bleeding, we should expect effects in the same direction, if not of a similar magnitude.

Table 1 Baseline characteristics of participants prior to randomisation

\begin{tabular}{|c|c|c|}
\hline & $\begin{array}{l}\text { Tranexamic } \\
\text { acid }\end{array}$ & Placebo \\
\hline & $(n=10,000)$ & $(n=10,000)$ \\
\hline \multicolumn{3}{|l|}{ Age at randomisation (years): } \\
\hline$<16$ & $N(X \%)$ & $N(X \%)$ \\
\hline $16-25$ & N NNN (X \%) & N NNN (X \%) \\
\hline $26-33$ & N NNN (X \%) & N NNN (X \%) \\
\hline $34+$ & N NNN (X \%) & N NNN (X \%) \\
\hline Baby delivered in this hospital & N NNN (X \%) & N NNN (X \%) \\
\hline \multicolumn{3}{|l|}{ Type of delivery: } \\
\hline Vaginal & N NNN (X \%) & N NNN (X \%) \\
\hline Caesarean section & N NNN (X \%) & N NNN (X \%) \\
\hline
\end{tabular}

Time between delivery and randomisation (hours):

$\leq 1$

$>1$ to $\leq 3$

$>3^{\mathrm{a}}$

Placenta fully delivered:

$$
\text { Yes }
$$

No

Primary cause of haemorrhage:

Uterine atony

Placenta praevia/accreta

Surgical trauma/tears

Other

Unknown

Systolic BP (mmHg):

\begin{tabular}{|c|c|}
\hline$\geq 90$ & N NNN (X \%) \\
\hline
\end{tabular}

Estimated volume of blood lost $(\mathrm{mL})$ :

$$
\leq 500
$$

$>500$ to $\leq 1000$

$>1000$

Uterotonic prophylaxis given:

Yes

No

Not known

Clinical signs of haemodynamic instability:

\begin{tabular}{lrl} 
Yes & $N$ NNN $(X \%)$ & $N$ NNN $(X \%)$ \\
No & $N$ NNN $(X \%)$ & N NNN (X \%) \\
\hline Includes $X$ patients randomly assigned more than $24 \mathrm{~h}$ after delivery
\end{tabular}

During the trial, site monitoring showed that some hysterectomies are conducted before randomisation. For example, in response to the onset of severe lifethreatening bleeding during caesarean section, an 
obstetrician may decide to conduct a hysterectomy, and while the hysterectomy is underway, the woman is enrolled into the trial. Similarly, in some cases of uterine rupture, a hysterectomy is sometimes done prior to randomisation. In other cases, a hysterectomy was conducted in a peripheral hospital, and the woman is enrolled into the WOMAN trial after having been transferred for the management of ongoing bleeding. Although TXA might reduce the risk of death in these cases, it could not affect the risk of hysterectomy because the hysterectomy was carried out prior to randomisation. Including such patients in the analysis will dilute the treatment effect towards the null. Therefore, only hysterectomies carried out after randomisation will be included. All hysterectomies will be monitored before database lock using the Primary Outcome Monitoring Form to confirm which ones were done after randomisation (see Additional file 2).

Pearson's chi-squared test will be used as the primary test of statistical significance of the effect of treatment allocation on the composite of death or hysterectomy by 42 days. Frequencies and percentages per arm, and the risk ratio for the outcome with the treatment with its $95 \%$ confidence interval (CI) will be reported as shown in Table 2.

\section{Imputation of missing data}

As loss to follow-up is expected to be minimal (i.e. less than $1 \%$ missing data on the primary outcome), missing values will not be imputed.

\section{Subgroup analyses}

All subgroups will be defined according to variables that are measured before randomisation and will be presented as shown in Table 3. The following subgroup analyses will be carried out for the primary outcome (death or hysterectomy), unless otherwise stated.

\section{Time to treatment}

The WOMAN trial was planned before the results of the CRASH-2 trial of TXA in bleeding trauma patients were available. The CRASH-2 trial, which recruited 20,210 adults with significant traumatic bleeding, showed that TXA reduces death due to bleeding $(\mathrm{RR}=0.85,95 \% \mathrm{CI}$ $0.76-0.96)$ and all-cause mortality ( $R R=0.91,95 \% \mathrm{CI}$ 0.85-0.97), with no increase in vascular occlusive events. The CRASH-2 trial investigators hypothesised that TXA would be most effective when given soon after injury, when bleeding is profuse, but less effective later, when the acute phase response to trauma increases the risk of thrombosis. They tested this hypothesis by conducting a pre-specified subgroup analysis of the effect of TXA by time from injury to the start of treatment $(\leq 1$, $1-\leq 3,3-8 \mathrm{~h}$ ) and found strong evidence in support of their hypothesis (test of homogeneity $p<0.0001$ ). In patients treated within $3 \mathrm{~h}$ of injury, TXA substantially reduced the risk of death due to bleeding, but when given after $3 \mathrm{~h}$, TXA appeared to increase the risk of death due to bleeding [6].

Early activation of fibrinolysis is common after trauma and is associated with increased mortality [14]. Tissue plasminogen activator (TPA), the enzyme that converts plasminogen to the active fibrinolytic enzyme plasmin, is stored within the vascular endothelium in secretory organelles called Weibel Palade bodies. Trauma triggers the rapid release of tissue plasminogen activator (TPA) from the vascular endothelium resulting in fibrinolysis and increased bleeding [15]. By inhibiting early fibrinolysis, TXA reduces the risk of exsanguination. The apparent increase in death due to bleeding with late TXA administration may reflect PAI-1-induced suppression of fibrinolysis and the onset of thrombotic disseminated intravascular coagulation (DIC) [16]. The latter is characterised by intravascular activation of coagulation with widespread fibrin deposition. By inhibiting fibrinolysis, TXA could worsen thrombotic DIC. Although the underlying pathology is thrombotic, due to the consumption of coagulation factors, thrombotic DIC usually manifests as bleeding.

Table 2 Effect of tranexamic acid (TXA) on death or hysterectomy

\begin{tabular}{|c|c|c|c|c|}
\hline & $\begin{array}{l}\text { TXA } \\
(n=10,000)\end{array}$ & $\begin{array}{l}\text { Placebo } \\
(n=10,000)\end{array}$ & Risk ratio (95 \% Cl) & $p$ value* \\
\hline Death or hysterectomy & NN (X \%) & NN (X \%) & $X . X X(X . X X-X . X X)$ & $p=0 . X X$ \\
\hline Death (all causes) & NN (X \%) & NN (X \%) & $X . X X(X . X X-X . X X)$ & $p=0 . X X$ \\
\hline Bleeding deaths & NN (X \%) & NN (X \%) & $X . X X(X . X X-X . X X)$ & $p=0 . X X$ \\
\hline Disseminated intravascular coagulation deaths & NN (X \%) & NN (X \%) & $X . X X(X . X X-X . X X)$ & $p=0 . X X$ \\
\hline Pulmonary embolic deaths & NN (X \%) & NN (X \%) & $X . X X(X . X X-X . X X)$ & $p=0 . X X$ \\
\hline Sepsis deaths & NN (X \%) & NN (X \%) & $X . X X(X . X X-X . X X)$ & $p=0 . X X$ \\
\hline Deaths due to other causes & NN (X \%) & NN (X \%) & $X . X X(X . X X-X . X X)$ & $p=0 . X X$ \\
\hline Hysterectomy & NN (X \%) & NN (X \%) & X.XX (X.XX-X.XX) & $p=0 . X X$ \\
\hline
\end{tabular}

${ }^{*} p$ values from Pearson's chi-squared test 
Table 3 Effect of tranexamic acid (TXA) on death or hysterectomy by subgroups

\begin{tabular}{|c|c|c|c|c|c|}
\hline \multirow[t]{2}{*}{ Subgroup } & \multicolumn{2}{|l|}{ TXA } & \multicolumn{2}{|l|}{ Placebo } & \multirow[t]{2}{*}{ Risk ratio $(99 \% \mathrm{Cl})$} \\
\hline & Death or hysterectomy & Received TXA & Death or hysterectomy & Received Placebo & \\
\hline \multicolumn{6}{|c|}{ Time between delivery and randomisation: } \\
\hline$\leq 1 \mathrm{~h}$ & NN (X \%) & N NNN & NN (X \%) & N NNN & $X . X X(X . X X-X . X X)$ \\
\hline $1-3 h$ & NN (X \%) & N NNN & NN (X \%) & N NNN & $X . X X(X . X X-X . X X)$ \\
\hline$>3 h$ & NN (X \%) & N NNN & NN (X \%) & N NNN & $X . X X(X . X X-X . X X)$ \\
\hline \multicolumn{6}{|l|}{$(p=0 . X X X)^{*}$} \\
\hline \multicolumn{6}{|l|}{ Type of delivery: } \\
\hline Vaginal & NN (X \%) & N NNN & NN (X \%) & N NNN & $X . X X(X . X X-X . X X)$ \\
\hline Caesarean section & NN (X \%) & N NNN & NN (X \%) & N NNN & $X . X X(X . X X-X . X X)$ \\
\hline \multicolumn{6}{|l|}{$(p=0 . X X X)$} \\
\hline \multicolumn{6}{|c|}{ Primary cause of haemorrhage: } \\
\hline Uterine atony & NN (X \%) & N NNN & NN (X \%) & N NNN & $X . X X(X . X X-X . X X)$ \\
\hline Other/unknown & NN (X \%) & N NNN & NN (X \%) & N NNN & $X . X X(X . X X-X . X X)$ \\
\hline$(p=0 . X X X)$ & & & & & \\
\hline
\end{tabular}

Similar temporal changes in fibrinolysis have been observed after childbirth [17]. Within $1 \mathrm{~h}$ of delivery, the serum concentration of TPA doubles, possibly due to the trauma of childbirth. Thereafter, the TPA concentration falls steeply. On the other hand, levels of the plasminogen activator inhibitors (PAI-1 and PAI-2) are increased around the time of delivery and remain so for several days. We might therefore expect TXA would be most effective when given soon after delivery, when TPA levels are highest, and less effective (possibly even harmful) when given several hours after delivery, when the risk of thrombotic DIC may be increased. We will examine this hypothesis by conducting a sub-group analysis of the effect of TXA according to the time interval between delivery and TXA treatment $(\leq 1,>1$ to $\leq 3,>3 \mathrm{~h})$. The outcome measure for this subgroup analysis will be death due to bleeding. The results of this analysis will be considered in the context of the relevant biological and clinical data on the time to treatment interaction.

\section{Type of delivery}

Because a substantial proportion of all deliveries are by caesarean section and caesarean section is an established risk factor for post-partum haemorrhage [18], it is important to examine whether the effect of TXA on the risk of death and hysterectomy varies by type of delivery. We do not anticipate substantial heterogeneity by type of delivery.

\section{Primary cause of post-partum haemorrhage}

For post-partum haemorrhage due to uterine atony, uterotonics are recommended as the first line treatment [19]. It is believed that uterine contraction will compress the spiral arteries and reduce bleeding. When planning the WOMAN trial, some reviewers expressed concern that administration of the WOMAN trial treatment might lead to fewer women receiving prophylactic uterotonics. For this reason, we collected information on uterotonic use and planned a sub-group analysis on the basis of whether or not uterotonics were administered. It was suggested that TXA would be less effective in women who receive uterotonics, particularly when uterine atony is the cause of bleeding. However, because $99 \%$ of women recruited to May 2014 received uterotonics, the planned subgroup analysis would be uninformative. Instead, we will examine the effect of TXA by cause of the bleeding (uterine atony versus all other causes) as determined at baseline. We do not anticipate substantial heterogeneity by cause of haemorrhage.

The main analysis for the pre-specified subgroups will be an unadjusted test of interaction in a logistic regression model to assess evidence for whether the effect of treatment differs across subgroup categories. Unless there is strong evidence against the null hypothesis of homogeneity of effects (i.e. $p<0.001$ ), the overall relative risk will be considered the most reliable guide to the approximate relative risks in all subgroups. For each subgroup, we will report frequencies and percentages per arm with the RR and $99 \% \mathrm{CI}$ as estimates of stratum-specific treatment effects, with the $p$ value for the test of interaction.

\section{Secondary outcomes \\ Death}

Death will be analysed (separately from hysterectomy) as a secondary outcome. As outlined above, although the use of a composite endpoint increases the statistical power, 
if the effect of TXA on death and hysterectomy are not similar, it could make interpretation of the primary results difficult. The causes of death will be analysed as shown in Table 2. Also, there is no reason to believe that TXA can prevent deaths from causes such as eclampsia or sepsis, unless death from these other causes is made more likely by bleeding. Therefore, we will assess the effect of TXA on all-cause mortality and cause-specific mortality (death due to bleeding, pulmonary embolism and other causes (Table 2). We will present the distribution of causes of death by days since randomisation as described in Fig. 2 .

\section{Thromboembolic events}

An increased risk of venous thromboembolic events (VTE) persists for several weeks post-partum [20]. Venous thrombosis is one of the leading causes of maternal morbidity and mortality with about $95 \%$ of these events occurring during the first 4 weeks post-partum [21]. The risk of deep vein thrombosis (DVT) is believed to be higher than pulmonary embolism (PE) in the postpartum period. Any further increase in risk from TXA use would be important. We will therefore assess and present the risk of venous (DVT and PE) and arterial events (myocardial infarction (MI) and stroke) as shown in Table 4. The definitions of DVT, PE, stroke and MI used in the trial are shown in Additional file 3.

\section{Surgical and radiological interventions to treat haemorrhage}

Hysterectomy will be analysed as a secondary outcome (Table 2). Additionally, the following interventions will be analysed: manual removal of the placenta, intrauterine tamponade, embolisation, brace sutures of the uterus, arterial ligation and laparotomy for other reasons (carried out after randomisation to control bleeding and achieve haemostasis for procedures other than those listed). If TXA reduces bleeding, it may also reduce the need for these additional treatments. However, as the decision to randomise can be made at the same time, or even after these interventions are carried out, some dilution of the treatment effect towards the null might be expected. Surgical and radiological events will be analysed in two ways: as individual events and grouped as 'surgical and radiological events'. These will be summarised as the number and percentage of patients per arm. These will be presented as shown in Table 4 .

\section{Blood transfusion}

TXA has been shown to reduce the probability of receiving a blood transfusion by one-third in patients undergoing routine surgery [4]. If TXA can reduce the need for blood product transfusion in women with $\mathrm{PPH}$, this would be an important finding. We will therefore assess the effect of TXA on the use of whole blood or packed red blood cells, fresh frozen plasma and other blood products in transfusion. These will be summarised as the number and percentage of patients per arm who receive these, and the mean, (SD) or median (range) as appropriate, units transfused per treatment arm. The effect of treatment allocation will be tested using a two-sample $t$ test (or Mann-Whitney rank sum test, as appropriate). However, once again, since the decision to transfuse is often made before or at the time of randomisation, some dilution towards the null is to be expected. The results will be presented as shown in Table 4 .

\section{Number of deaths}

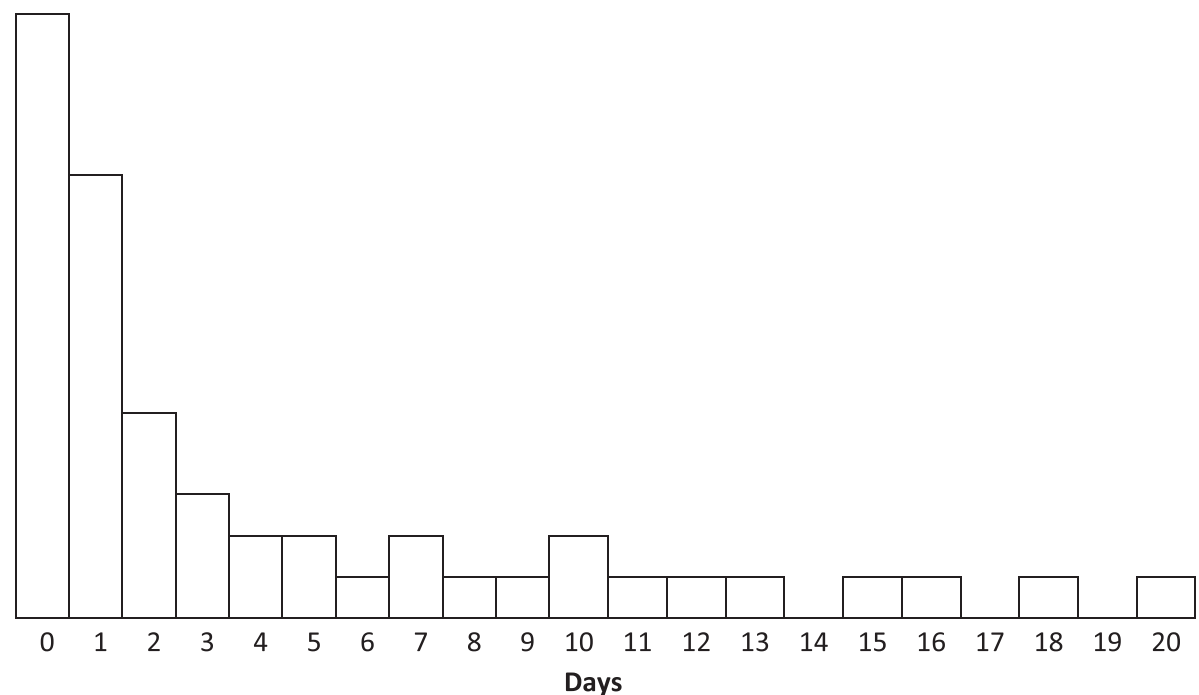

Fig. 2 Distribution of cause of death by days since randomisation. Notes: Bars will be stacked to show number of deaths due to bleeding, pulmonary embolism, or other causes. 
Table 4 Effect of tranexamic acid (TXA) on thromboembolic events, need for surgery and transfusion and level of dependency

\begin{tabular}{|c|c|c|c|c|}
\hline \multirow{3}{*}{ Thromboembolic events } & \multirow{2}{*}{$\begin{array}{l}\text { TXA } \\
(n=10,000)\end{array}$} & \multirow{2}{*}{$\begin{array}{l}\text { Placebo } \\
(n=10,000)\end{array}$} & \multirow[t]{2}{*}{ Risk ratio (95 \% Cl) } & \multirow[t]{2}{*}{$p$ value $^{*}$} \\
\hline & & & & \\
\hline & & & & \\
\hline Any event & NN (X \%) & NN (X \%) & $X . X X(X . X X-X . X X)$ & $p=0 . X X$ \\
\hline Venous events (DVT, PE) & NN (X \%) & NN (X \%) & $X . X X(X . X X-X . X X)$ & $p=0 . X X$ \\
\hline DVT & NN (X \%) & NN (X \%) & $X . X X(X . X X-X . X X)$ & $p=0 . X X$ \\
\hline PE & NN (X \%) & NN (X \%) & X.XX (X.XX-X.XX) & $p=0 . X X$ \\
\hline Arterial events (MI, stroke) & NN (X \%) & NN (X \%) & X.XX (X.XX-X.XX) & $p=0 . X X$ \\
\hline $\mathrm{Ml}$ & NN (X \%) & NN (X \%) & X.XX (X.XX-X.XX) & $p=0 . X X$ \\
\hline Stroke & NN (X \%) & NN (X \%) & $X . X X(X . X X-X . X X)$ & $p=0 . X X$ \\
\hline \multicolumn{5}{|l|}{ Surgical interventions } \\
\hline Hysterectomy & NN (X \%) & NN (X \%) & X.XX (X.XX-X.XX) & $p=0 . X X$ \\
\hline Manual removal of placenta & NN (X \%) & NN (X \%) & $X . X X(X . X X-X . X X)$ & $p=0 . X X$ \\
\hline Intrauterine tamponade & NN (X \%) & NN (X \%) & X.XX (X.XX-X.XX) & $p=0 . X X$ \\
\hline Embolisation & NN (X \%) & NN (X \%) & $X . X X(X . X X-X . X X)$ & $p=0 . X X$ \\
\hline Brace sutures of uterus & NN (X \%) & NN (X \%) & X.XX (X.XX-X.XX) & $p=0 . X X$ \\
\hline Arterial ligation & NN (X \%) & NN (X \%) & $X . X X(X . X X-X . X X)$ & $p=0 . X X$ \\
\hline Laparotomy & NN (X \%) & NN (X \%) & $X . X X(X . X X-X . X X)$ & $p=0 . X X$ \\
\hline Any surgical or radiological intervention & NN (X \%) & NN (X \%) & $X . X X(X . X X-X . X X)$ & $p=0 . X X$ \\
\hline \multicolumn{5}{|l|}{ Blood transfusion } \\
\hline Whole blood & NN (X \%) & NN (X \%) & X.XX (X.XX-X.XX) & $p=0 . X X$ \\
\hline Packed red blood cells & NN (X \%) & NN (X \%) & $X . X X(X . X X-X . X X)$ & $p=0 . X X$ \\
\hline Frozen plasma & NN (X \%) & NN (X \%) & X.XX (X.XX-X.XX) & $p=0 . X X$ \\
\hline Other blood products & NN (X \%) & NN (X \%) & $X . X X(X . X X-X . X X)$ & $p=0 . X X$ \\
\hline Any transfusion & NN (X \%) & NN (X \%) & X.XX (X.XX-X.XX) & $p=0 . X X$ \\
\hline \multicolumn{5}{|l|}{ Blood transfusion (units) } \\
\hline Whole blood & mean (SD) & mean (SD) & Diff in means $(X . X-X . X)$ & $p=0 . X X$ \\
\hline Packed red blood cells & mean (SD) & mean (SD) & Diff in means $(X . X-X . X)$ & $p=0 . X X$ \\
\hline Frozen plasma & mean (SD) & mean (SD) & Diff in means $(X . X-X . X)$ & $p=0 . X X$ \\
\hline Other blood products & mean (SD) & mean (SD) & Diff in means $(X . X-X . X)$ & $p=0 . X X$ \\
\hline Any transfusion & mean (SD) & mean (SD) & Diff in means $(X . X-X . X)$ & $p=0 . X X$ \\
\hline \multicolumn{5}{|l|}{$E Q-5 D^{a}$} \\
\hline Mobility & NN (X \%) & NN (X \%) & X.XX (X.XX-X.XX) & $p=0 . X X$ \\
\hline Self-care & NN (X \%) & NN (X \%) & X.XX (X.XX-X.XX) & $p=0 . X X$ \\
\hline Usual activities & NN (X \%) & NN (X \%) & X.XX (X.XX-X.XX) & $p=0 . X X$ \\
\hline Pain/discomfort & NN (X \%) & NN (X \%) & X.XX (X.XX-X.XX) & $p=0 . X X$ \\
\hline Anxiety/depression & NN (X \%) & NN (X \%) & X.XX (X.XX-X.XX) & $p=0 . X X$ \\
\hline Visual analogue scale & mean (SD) & mean (SD) & Diff in means $(X . X-X . X)$ & $p=0 . X X$ \\
\hline
\end{tabular}

$D V T$ deep vein thrombosis, $M I$ myocardial infarction, $P E$ pulmonary embolism

${ }^{*} p$ values from Pearson's chi-squared test

aproportion with severe problems

\section{Quality of life}

The impact on the quality of life of women who suffer $\mathrm{PPH}$ is not well researched. One study suggests that the health and quality of life of women after maternal morbidities such as $\mathrm{PPH}$, negatively affects the woman's wellbeing and quality of life [22]. Information on the health status of women is collected on the outcome form using the EQ-5D. This consists of two principal measurement components. The first defines healthrelated quality of life in terms of five dimensions: 
'mobility', 'self-care,' 'usual activities', 'pain/discomfort' and 'anxiety/depression' [23]. Responses in each dimension are divided into three ordinal levels, coded: (1) no problems, (2) some or moderate problems and (3) severe or extreme problems. The second measurement component of the EQ-5D consists of a $20-\mathrm{cm}$ vertical visual analogue scale ranging from 100 (best imaginable health state) to 0 (worst imaginable health state), which provides an indication of the woman's health status. EQ-5D Scores will be presented as the proportions of women reporting problems (i.e. some, moderate, severe or extreme) on each EQ$5 \mathrm{D}$ dimension. In addition, the means (with SD) of the EQ-5D visual analogue scales per arm and the difference in the means will also be reported in order to assess the treatment effect, along with the $p$ value from a twosample $t$-test. These will be presented as shown in Table 4 .

\section{Complications}

Women with PPH are at risk of other significant medical events associated with pregnancy and $\mathrm{PPH}$. These include renal failure, cardiac failure, respiratory failure, hepatic failure, sepsis and seizure, and these events are collected routinely as outcomes. An increase in the risk of seizure using high doses of TXA has been reported $[24,25]$. These will be presented as shown in Table 5 .

\section{Adverse events}

Other untoward medical events are collected up to 42 days after randomisation as adverse events (AEs). In line with ICH-GCP guidelines [26], an AE is considered as serious if it results in death, is life-threatening, requires inpatient hospitalisation or prolongation of existing hospitalisation, results in persistent or significant disability/incapacity or results in a congenital anomaly/ birth defect. Where there is at least a possibility of an adverse event being causally linked to the trial drug, this is considered to be an adverse reaction. A suspected unexpected serious adverse reaction (SUSAR) is an unexpected occurrence of a serious adverse reaction. There need only to be an index of suspicion that the event is a previously unreported reaction to a trial drug or a previously reported but exaggerated or unexpectedly frequent adverse drug reaction.

The number of AEs, SAEs, and SUSARs grouped by MedDRA $^{\circ}$ codes [27] and the number of patients with at least one event will be compared between arms using a chisquared test (or Fisher's exact test), with RRs and $95 \% \mathrm{CI}$ when these are computable. Frequencies and percentages per treatment group will be presented as shown in Table 6 .

\section{Status of babies}

TXA is believed to pass into breast milk to a concentration of approximately one hundredth of the concentration in the maternal blood [28]. The number and percentage of babies born alive per treatment group will be described. Where babies were born alive and breastfed during the course of the follow-up period, their status (alive or dead) will be presented as shown in Table 7 .

\section{Thromboembolic events in breastfed babies}

Data on any thromboembolic events (TE) in babies are collected. TE was defined as follows: the event must be a confirmed diagnosis and may include any venous or arterial thrombosis (thrombosis of limb artery/deep veins, renal artery/veins, pulmonary embolism, hepatic veins, caval veins, intracardiac thrombosis, portal vein, mesenteric veins/artery, cerebral veins, retinal vein, ischemic stroke, arteries, aorta, myocardial infarction, microvascular thrombosis from purpura fulminans or disseminated intravascular coagulation. These data will be presented as shown in Table 7.

For all events reported in Tables 4, 5, 6 and 7, frequencies and percentages per arm will be presented, with a $\mathrm{RR}$ and $95 \% \mathrm{CI}$ as estimates of treatment effect, along with the $p$ value from Pearson's chi-squared test.

\section{Other analyses: to be reported in a separate publication}

Analysis 1: Adjusting for possible imbalance in baseline prognostic factors

In a large trial such as the WOMAN trial, baseline characteristics of patients that may influence the outcome

Table 5 Complications

\begin{tabular}{|c|c|c|c|c|}
\hline $\begin{array}{l}\text { Type of adverse } \\
\text { event }\end{array}$ & $\begin{array}{l}\text { Tranexamic acid } \\
(\mathrm{n}=10,000)\end{array}$ & $\begin{array}{l}\text { Placebo } \\
(n=10,000)\end{array}$ & Risk ratio $(95 \% \mathrm{Cl}$ ) & $p$ value* $^{*}$ \\
\hline Renal failure & $N(X \%)$ & $N(X \%)$ & X.XX (X.XX-X.XX) & $p=0 . X X$ \\
\hline Cardiac failure & $N(X \%)$ & $N(X \%)$ & X.XX (X.XX-X.XX) & $p=0 . X X$ \\
\hline Respiratory failure & $N(X \%)$ & $N(X \%)$ & X.XX (X.XX-X.XX) & $p=0 . X X$ \\
\hline Hepatic failure & $N(X \%)$ & $N(X \%)$ & X.XX (X.XX-X.XX) & $p=0 . X X$ \\
\hline Sepsis & $N(X \%)$ & $N(X \%)$ & X.XX (X.XX-X.XX) & $p=0 . X X$ \\
\hline Seizure & $N(X \%)$ & $N(X \%)$ & $X . X X(X . X X-X . X X)$ & $p=0 . X X$ \\
\hline
\end{tabular}


Table 6 Adverse events

\begin{tabular}{|c|c|c|c|c|}
\hline $\begin{array}{l}\text { Type of adverse } \\
\text { event }^{\mathrm{a}}\end{array}$ & $\begin{array}{l}\text { Tranexamic acid } \\
(n=10,000)\end{array}$ & $\begin{array}{l}\text { Placebo } \\
(n=10,000)\end{array}$ & $\begin{array}{l}\text { Risk ratio } \\
(95 \% \mathrm{Cl})\end{array}$ & $p$ value* \\
\hline MedDRA code groups & N (X \%) & $N(X \%)$ & $X . X X(X . X X-X . X X)$ & $p=0 . X X$ \\
\hline
\end{tabular}

should be evenly distributed between the treatment and placebo groups, so that any difference in outcome can be attributed to the intervention. However, it is still possible that a chance imbalance in important prognostic factors could influence the results $[29,30]$. To investigate this possibility, we will conduct an analysis of the effect of treatment that is adjusted for baseline risk. We will build a prognostic model based on pre-specified baseline variables and use it to estimate the predicted risk of the outcome at baseline.

The primary outcome is death or hysterectomy; death due to bleeding is an important secondary outcome. The most important prognostic factors for these outcomes that are measured at baseline are maternal age, estimated blood loss, systolic blood pressure and haemodynamic instability. These variables will be included in a multivariable prognostic model based on the final trial dataset. Although there are almost complete data on these variables, in the case of missing data, the missing values will be replaced by the mean of the observed data [31].

The trial data will then be stratified into risk deciles as shown in Tables 8 and 9 based on the predicted risk of the outcomes at baseline. We will report frequencies and percentages within each risk decile, and calculate a risk ratio (with $95 \% \mathrm{CI}$ ) for each risk decile. The pooled risk ratio (with a $95 \% \mathrm{CI}$ ) will be estimated as an inverse variance weighted average of the stratum-specific risk ratios. The pooled risk ratio should provide an estimate of the treatment effect that is un-confounded by baseline risk. The advantage of this approach is that the effect of baseline risk on the treatment effect is more explicit than when covariate-adjusted odds ratios are calculated using logistic regression. Furthermore, risk ratios are easier to interpret and apply to individual patients than are odds ratios [32].

A forest plot will be prepared to show graphically how the treatment effect varies by baseline risk. We will use a chi-squared test to assess any heterogeneity in the treatment effect across the risk groups, and we will calculate the I-squared statistic to quantify the percentage of variability in effect estimates that is due to heterogeneity rather than chance. To reduce the likelihood of making inappropriate inferences, we pre-specify that unless there is strong evidence against the null hypothesis of homogeneity of effects (i.e. $p<0.001$ ), the pooled relative risk will be considered the most reliable guide to the approximate treatment effects in all risk strata. We do not anticipate substantial heterogeneity by baseline risk. However, we hypothesise that the risk reduction with TXA would be greatest in patients at low risk of death since a smaller proportion of these women will have unsurvivable bleeding.

\section{Analysis 2: Cost-effectiveness}

An economic analysis will be relevant if TXA clearly demonstrates efficacy in achieving its clinical aims. In this case, the study will be undertaken in the form of a cost-effectiveness analysis with the aim of estimating the incremental cost-effectiveness ratio comparing the use of TXA with normal clinical practice. Analysis will be based on adjusted life years gained. A further analysis will explore the use of the EQ-5D data to qualityadjusted survival. In this study, the economic analysis is clearly bounded as virtually all significant resource use will occur in the initial period of hospitalisation. As such, neither a long-term resource analysis nor an analysis of out-of-hospital costs will be required. The trial use of TXA is likely to mirror its use in normal clinical practice, hence the cost-effectiveness estimated in the trial (adjusted for protocol driven costs) will closely approximate cost-effectiveness in actual clinical practice. Data on physical resource consumption (e.g. the length and nature of hospital stay) will be collected for each patient and a common unit cost at a country level will be

Table 7 Death or thromboembolic events in breast-fed babies ${ }^{a}$

\begin{tabular}{|c|c|c|c|c|}
\hline & $\begin{array}{l}\text { Tranexamic acid } \\
(n=10,000)\end{array}$ & $\begin{array}{l}\text { Placebo } \\
(n=10,000)\end{array}$ & $\begin{array}{l}\text { Risk ratio } \\
(95 \% \mathrm{Cl})\end{array}$ & $p$ value $^{*}$ \\
\hline Any death (of breastfed baby) & NN (X \%) & NN (X \%) & $X . X X(X . X X-X . X X)$ & $p=0 . X X$ \\
\hline Any thromboembolic event (of breastfed baby) & NN (X\%) & NN (X\%) & $X . X X(X . X X-X . X X)$ & $p=0 . X X$ \\
\hline
\end{tabular}

${ }^{*} p$ values from Pearson's chi-squared test

${ }^{a}$ Babies born alive and breastfed for some duration 
Table 8 Death or hysterectomy by predicted risk at baseline

\begin{tabular}{|c|c|c|c|c|c|}
\hline \multirow[t]{2}{*}{ Risk decile $^{a}$} & \multicolumn{2}{|l|}{ Tranexamic acid (TXA) } & \multicolumn{2}{|l|}{ Placebo } & \multirow[t]{2}{*}{ Risk ratio $(95 \% \mathrm{Cl})$} \\
\hline & Death or hysterectomy & Received TXA & Death or hysterectomy & Received placebo & \\
\hline 1 & NN (X \%) & N NNN & NN (X \%) & N NNN & X.XX (X.XX-X.XX) \\
\hline 2 & NN (X \%) & N NNN & NN (X \%) & N NNN & X.XX (X.XX-X.XX) \\
\hline 3 & NN (X \%) & N NNN & NN (X \%) & N NNN & X.XX (X.XX-X.XX) \\
\hline 4 & NN (X \%) & N NNN & NN (X \%) & N NNN & X.XX (X.XX-X.XX) \\
\hline 5 & NN (X \%) & N NNN & NN (X \%) & N NNN & $X . X X(X . X X-X . X X)$ \\
\hline 6 & NN (X \%) & N NNN & NN (X \%) & N NNN & X.XX (X.XX-X.XX) \\
\hline 7 & NN (X \%) & N NNN & NN (X \%) & N NNN & $X . X X(X . X X-X . X X)$ \\
\hline 8 & NN (X \%) & N NNN & NN (X \%) & N NNN & X.XX (X.XX-X.XX) \\
\hline 9 & NN (X \%) & N NNN & NN (X \%) & N NNN & X.XX (X.XX-X.XX) \\
\hline 10 & NN (X \%) & N NNN & NN (X \%) & N NNN & X.XX (X.XX-X.XX) \\
\hline Overall & NN (X \%) & N NNN & NN (X \%) & N NNN & X.XX (X.XX-X.XX) \\
\hline$\left(P^{2}=X \%, p=0 . X X\right)$ & & & & & \\
\hline
\end{tabular}

applied. A sensitivity analysis will be undertaken to assess the robustness of the economic analysis in response to variations in key variables such as drug prices. In all cases, the economic analysis will be integrated with the clinical trial procedures to optimise efficiency and minimise inconvenience to patients.

Mechanical ventilation and intensive care unit (ICU) admission are resource consumption measures and are not required for all patients. Mechanical ventilation will be summarised as number and percentage of patients per arm who received such a therapy. Where mechanical ventilation is used exclusively for general anaesthetic for surgery, this data is excluded. Length of stay in the ICU and the hospital will be censored due to early deaths or by a stay in the ICU or hospital longer than 42 days. Summary statistics will include the median and the interquartile range computed separately for each treatment arm.

\section{Data monitoring and interim analyses}

An independent Data Monitoring Committee (DMC) is responsible for reviewing the progress of the trial, including recruitment, data quality and main outcomes and safety data. The DMC has the responsibility for

Table 9 Death due to bleeding by predicted risk at baseline

\begin{tabular}{|c|c|c|c|c|c|}
\hline \multirow[t]{2}{*}{ Risk decile ${ }^{a}$} & \multicolumn{2}{|l|}{ Tranexamic acid (TXA) } & \multicolumn{2}{|l|}{ Placebo } & \multirow[t]{2}{*}{ Risk ratio $(95 \% \mathrm{Cl})$} \\
\hline & Death due to bleeding & Received TXA & Death due to bleeding & Received placebo & \\
\hline 1 & NN (X \%) & N NNN & NN (X \%) & N NNN & $X . X X(X . X X-X . X X)$ \\
\hline 2 & NN (X \%) & N NNN & NN (X \%) & N NNN & $X . X X(X . X X-X . X X)$ \\
\hline 3 & NN (X \%) & N NNN & NN (X \%) & N NNN & $X . X X(X . X X-X . X X)$ \\
\hline 4 & NN (X\%) & N NNN & NN (X \%) & N NNN & $X . X X(X . X X-X . X X)$ \\
\hline 5 & NN (X\%) & N NNN & NN (X \%) & N NNN & $X . X X(X . X X-X . X X)$ \\
\hline 6 & NN (X \%) & N NNN & NN (X \%) & N NNN & $X . X X(X . X X-X . X X)$ \\
\hline 7 & NN (X \%) & N NNN & NN (X \%) & N NNN & $X . X X(X . X X-X . X X)$ \\
\hline 8 & NN (X \%) & N NNN & NN (X \%) & N NNN & $X . X X(X . X X-X . X X)$ \\
\hline 9 & NN (X \%) & N NNN & NN (X \%) & N NNN & $X . X X(X . X X-X . X X)$ \\
\hline 10 & NN (X \%) & N NNN & NN (X \%) & N NNN & $X . X X(X . X X-X . X X)$ \\
\hline Overall & NN (X \%) & N NNN & NN (X \%) & N NNN & $X . X X(X . X X-X . X X)$ \\
\hline$\left(P^{2}=X \%, p=0 . X X\right)$ & & & & & \\
\hline
\end{tabular}


deciding whether, while randomisation is in progress, the un-blinded results (or the un-blinded results for a particular subgroup), should be revealed to the TSC. They will do this if, and only if, two conditions are satisfied: (1) the results provide proof beyond reasonable doubt that treatment is on balance either definitely harmful or definitely favourable for all, or for a particular category of, participants in terms of the major outcome, and (2) the results, if revealed, would be expected to substantially change the prescribing patterns of clinicians who are already familiar with other trial results that exist. Exact criteria for 'proof beyond reasonable doubt' are not, and cannot be, specified by a purely mathematical stopping rule, but they are strongly influenced by such rules. This is in agreement with the Haybittle-Peto stopping rule [33, 34], whereby an interim analysis of major endpoint would generally need to involve a difference between treatment and control of at least three standard errors to justify premature disclosure. An interim subgroup analysis would have to be even more extreme to justify disclosure. This rule has the advantage that the exact number and timing of interim analyses need not be pre-specified. In summary, the stopping rules require extreme differences to justify premature disclosure and involve an appropriate combination of mathematical stopping rules and scientific judgment. To date, seven interim analyses by the DMC have been conducted with no recommendation for early stopping.

\section{Data management and analysis software}

The clinical database management system for WOMAN Trial was built to comply with ICH-GCP and uses MySQL and its accompanying manuals. PHP was used to develop the dynamic web pages for user interface. The database was developed by Sealed Envelope Ltd. Data are collected at each participating site and transmitted directly to the clinical trials unit (CTU) via the database. Where there is poor internet connection, the paper CRFs can be sent by fax or via email. Data checks and cleaning are performed by the CTU. Data items to be coded including Adverse Event term and terms used to describe 'other' causes of death on the Outcome Form are coded using MedDRA Version 12 [27]. The final database lock will take place at the end of the trial within 3 months from the time when the 'last patient' in the 'last follow-up' has completed the trial. Data will be exported for statistical analysis using the most recent version of Stata (StataCorp LP, College Station, Texas, USA).

\section{Data sharing}

Following publication of the primary and secondary analyses detailed in this statistical analysis plan, the trial data will be made available via our data sharing portal The Free Bank of Injury and emergency Research Data (freeBIRD) website (http://freebird.Lshtm.ac.uk). This will allow for maximum utilisation of the data to improve patient care and advance medical knowledge.

\section{Discussion}

This report presents and justifies the changes to our previously published protocol. The main changes are an increased sample size from 15,000 patients to 20,000 and changes to our planned subgroup analyses, time from delivery to randomisation is a new subgroup, and the removal of the subgroup 'use of uterotonic prophylaxis'.

We have presented our plan for the statistical analyses in advance of the database lock and un-blinding to guard against data dependent analyses.

The WOMAN trial will provide reliable evidence for the efficacy of tranexamic acid in the prevention of death, hysterectomy and other outcomes important to patients and clinicians. Aditional file 4 lists the collaborating investigators who have participated in the WOMAN Trial to date.

\section{Additional files}

Additional file 1: List of ethics approval for participating countries and sites. (DOCX $19 \mathrm{~kb}$ )

Additional file 2: Primary outcome monitoring form. (DOCX $34 \mathrm{~kb}$ )

Additional file 3: Guidance for diagnosis of complications. (DOCX 59 kb) Additional file 4: Trial collaborators. (DOCX 23 kb)

\section{Abbreviations}

AE: adverse event; Cl: confidence interval; CONSORT: Consolidated Standards of Reporting Trials; CRASH-2 trial: Clinical Randomisation of an Antifibrinolytic in Significant Haemorrhage Trial; CTU: clinical trials unit; DIC: disseminated intravascular coagulation; DMC: data monitoring committee; DVT: deep vein thrombosis; ICH-GCP: International Conference on Harmonisation of Technical Requirements for Registration of Pharmaceuticals for Human Use - Good Clinical Practice; ICU: intensive care unit; ITT: intention to treat; MySQL: My Structured Query Language; PAl: plasminogen activator inhibitors; PE: pulmonary embolism; PHP: PPH hypertext preprocessor; PPH: post-partum haemorrhage; SAE: serious adverse event;

SUSAR: suspected unexpected serious adverse reaction; SD: standard deviation; TE: thromboembolic events; TPA: tissue plasminogen activator; TXA: tranexamic acid; UK: United Kingdom; RR: relative risk; VTE: venous thromboembolic event; WOMAN: World Maternal Antifibrinolytic Trial.

\section{Competing interests}

The authors declare that they have no competing interests.

\section{Authors' contributions}

The WOMAN Trial was conceived by IR and HS, and all authors contributed to its design. This manuscript detailing the statistical plan was drafted by HS, $I R$ and PE. DE. ZA and CR reviewed and commented on it. All authors reviewed and agreed on the final manuscript.

\section{Acknowledgements}

The WOMAN Trial is funded by Department of Health (UK), grant number HICF-T2-0510-007; Wellcome Trust, grant number WT094947; and the Bill \& Melinda Gates Foundation, grant number OPP1095618. So far, the WOMAN 
Trial has recruited patients in 20 countries. We wish to thank all collaborators who have contributed to the trial so far (see Additional file 4).

The World Maternal Antifibrinolytic (WOMAN) trial aims to determine the effect of tranexamic acid (TXA) on death, hysterectomy, and other health outcomes in women with post-partum haemorrhage. It is an international, multicentre, randomised trial. We present a protocol update and the statistical analysis plan.

\section{Author details}

${ }^{1}$ Clinical Trials Unit, London School of Hygiene \& Tropical Medicine, Keppel Street, London WC1E 7HT, UK. ${ }^{2}$ Division of Perinatal and Reproductive Medicine, University of Liverpool, Liverpool Women's Hospital, Crown Street, Liverpool L8 7SS, UK. Infectious Diseases Epidemiology Unit, London School of Hygiene \& Tropical Medicine, Keppel Street, London WC1E 7HT, UK.

Received: 29 September 2015 Accepted: 6 April 2016

Published online: 17 May 2016

\section{References}

1. WHO, UNICEF, UNFPA, The World Bank. Trends in maternal mortality: 1990 to 2010. WHO, UNICEF, UNFPA, and The World Bank estimates. Geneva: World Health Organization; 2012

2. Say L, Chou D, Gemmill A, Tunçalp O, Moller A, Daniels J, et al. Global causes of maternal death: a WHO systematic analysis. Lancet Glob Health. 2014;2:e323-33.

3. Smith J, Mousa H. Peripartum hysterectomy for primary post-partum haemorrhage: incidence and maternal morbidity. J Obstet Gynaecol. 2007; 27:44-7.

4. Ker K, Prieto-Merino D, Roberts I. Systematic review, meta-analysis and meta-regression of the effect of tranexamic acid on surgical blood loss. Br J Surg. 2013;100(10):1271-9.

5. CRASH-2 trial collaborators, Shakur H, Roberts I, Bautista R, Caballero J, Coats $T$, et al. Effects of tranexamic acid on death, vascular occlusive events, and blood transfusion in trauma patients with significant haemorrhage (CRASH-2): a randomised, placebo-controlled trial. Lancet. 2010;376:23-32.

6. CRASH-2 collaborators, Roberts I, Shakur H, Afolabi A, Brohi K, Coats T, et al. The importance of early treatment with tranexamic acid in bleeding trauma patients: an exploratory analysis of the CRASH-2 randomised controlled trial. Lancet. 2011;377:1096-101.

7. Ducloy-Bouthors AS, Jude B, Duhamel A, Broisin F, Huissoud C, Keita-Meyer $\mathrm{H}$, et al. High-dose tranexamic acid reduces blood loss in postpartum haemorrhage. Crit Care. 2011;15(2):R117s.

8. Sentilhes L, Lasocki S, Ducloy-Bouthors AS, Deruelle P, Dreyfus M, et al. Tranexamic acid for the prevention and treatment of postpartum haemorrhage. Br J Anaesth. 2015;114(4):576-87.

9. Shakur H, Elbourne D, Gülmezoglu M, Alfirevic Z, Ronsmans C, Allen E, et al. The WOMAN trial (world maternal antifibrinolytic trial): tranexamic acid for the treatment of post-partum haemorrhage: an international randomized, double blind placebo controlled trial. Trials. 2010;11:40.

10. Schulz K, Altman D, Moher D. CONSORT 2010 Statement: updated guidelines for reporting parallel group randomised trials. BMC Med. 2010;8:18

11. Ferreira-Gonzalez I, Permanyer-Miralda G, Busse JW, et al. Methodologic discussions for using and interpreting composite endpoints are limited, but still identify major concerns. J Clin Epidemiol. 2007;60(7):651-7. 658-662.

12. Freemantle N, Calvert M, Wood J, Eastaugh J, Griffin C. Composite outcomes in randomized trials: greater precision but with greater uncertainty? JAMA. 2003;289(19):2554-9.

13. Tomlinson G, Detsky A. Composite end points in randomized trials: there is no free lunch. JAMA. 2010;303:267-8.

14. Sawamura A, Hayakawa M, Gando S, Kubota N, Sugano M, Wada T, et al. Disseminated intravascular coagulation with a fibrinolytic phenotype at an early phase of trauma predicts mortality. Thromb Res. 2009;1214:608-13.

15. Lowenstein CJ, Morrell CN, Yamakuchi M. Regulation of Weibel-Palade body exocytosis. Trends Cardiovasc Med. 2005;15:302-8.

16. Gando S, Otomo Y. Local hemostasis, immunothrombosis, and systemic disseminated intravascular coagulation in trauma and traumatic shock. Crit Care. 2015:19(1):72.
17. Kruithof E, Tran-Thang C, Gudinchet A, Hauert J, Nicoloso G, Genton C, et al. Fibrinolysis in pregnancy: a study of plasminogen activator inhibitors. Blood. 1987;69:460-6

18. Kramer MS, Berg C, Abenhaim H, Dahhou M, Rouleau J, Mehrabadi A, et al. Incidence, risk factors, and temporal trends in severe post-partum hemorrhage. Am J Obstet Gynecol. 2013;209:449.e1-7.

19. World Health Organization. WHO guidelines for the management of post-partum haemorrhage and retained placenta. Geneva: World Health Organization; 2009

20. Tepper NK, Boulet SL, Whiteman MK, Monsour M, Marchbanks PA, Hooper WC, et al. Post-partum venous thromboembolism: incidence and risk factors. Obstet Gynecol. 2014;123(5):987-96.

21. Pomp ER, Lenselink AM, Rosendaal FR, Doggen CJ. Pregnancy, the postpartum period and prothrombotic defects: risk of venous thrombosis in the MEGA study. J Thromb Haemost. 2008:6:632-7.

22. Waterstone M, Wolfe C, Hooper R, Bewley S. Postnatal morbidity after childbirth and severe obstetric morbidity. BJOG-Int J Obstet Gy. 2003;110: 128-33.

23. EuroQol Group. EuroQol - a new facility for the measurement of healthrelated quality of life. Health Pol. 1990;16:199-208.

24. Murkin JM, Falter F, Granton J, Young B, Burt C, Chu M. High-dose tranexamic acid is associated with nonischemic clinical seizures in cardiac surgical patients. Anesth Analg. 2010;110:350-3.

25. Furtmüller R, Schlag MG, Berger $M$, Hopf R, Huck S, Sieghart W, et al Tranexamic acid, a widely used antifibrinolytic agent, causes convulsions by a gamma-aminobutyric acid (A) receptor antagonistic effect. J Pharmacol Exp Ther. 2002;301:168-73.

26. $\mathrm{ICH}$. International Conference on Harmonisation $(\mathrm{ICH})$ harmonised tripartite guideline: guideline for good clinical practice (GCP). Geneva: ICH; 1996.

27. MedDRA ${ }^{\oplus}$ the Medical Dictionary for Regulatory Activities terminology is the international medical terminology developed under the auspices of the International Conference on Harmonisation of Technical Requirements for Registration of Pharmaceuticals for Human Use (ICH). www.meddra.org Accessed 11 May 2016.

28. Summary of Product Characteristics for Tranexamic Acid $100 \mathrm{mg} / \mathrm{m}$ Solution for Injection. https://www.medicines.org.uk/emc/medicine/28163 (Accessed 15 Sept 2015).

29. Pocock SJ, Assmann SE, Enos LE, Kasten LE. Subgroup analysis, covariate adjustment and baseline comparisons in clinical trial reporting: current practice and problems. Stat Med. 2002;21(19):2917-30.

30. Kahan B, Jairath $\mathrm{V}$, Doré $\mathrm{C}$, Morris T. The risks and rewards of covariate adjustment in randomized trials: an assessment of 12 outcomes from 8 studies. Trials. 2014;15:139.

31. White IR, Thompson SG. Adjusting for partially missing baseline measurements in randomized trials. Stat Med. 2005;24(7):993-1007.

32. Sackett DL, Deeks JJ, Altman D. Down with odds ratios! Evid-Based Med. 1996;1:164-7.

33. Haybittle UL. Repeated assessment of results in clinical trials of cancer treatment. Br J Radiol. 1971;44(526):793-7.

34. Peto R, Pike MC, Armitage P, Breslow NE, Cox DR, Howard SV, et al. Design and analysis of randomized clinical trials requiring prolonged observation of each patient. II. analysis and examples. Br J Cancer. 1977:35(1):1-39.

\section{Submit your next manuscript to BioMed Central and we will help you at every step:}

- We accept pre-submission inquiries

- Our selector tool helps you to find the most relevant journal

- We provide round the clock customer support

- Convenient online submission

- Thorough peer review

- Inclusion in PubMed and all major indexing services

- Maximum visibility for your research

Submit your manuscript at www.biomedcentral.com/submit 